\title{
Cervical abscess in an immunocompetent patient with Mycobacterium malmoense pulmonary disease
}

\author{
Joao N. Duarte $\cdot$ Nuno Marques $\cdot$ Leonor Barroso • \\ Isabel Ramos • Rosa Sá • David Sanz • Artur Ferreira • \\ Saraiva da Cunha
}

Received: 29 April 2011 / Accepted: 27 October 2011

(C) Springer-Verlag 2011
Keywords Mycobacterial cervical lymphadenitis . Disseminated atypical mycobacterial infection. Mycobacterium $\cdot$ Abscess

\section{Introduction}

Despite being a rare pathogen, Mycobacterium malmoense has been a subject of growing interest in recent decades, similar to what has been happening with other emerging nontuberculous mycobacteria (NTM).

M. malmoense is a slow-growing opportunistic and environmental pathogen, with no person-to-person transmission reported [1]. It was first described in 1977 by Schröder and Juhlin [2] in the city of Malmö, in Sweden, after being isolated from respiratory tract culture samples in four patients.

Since then, an increase in the number of isolates has been observed throughout the world, but especially in Northern Europe [3]. This increase was due not only to an improvement in culture methods and DNA identification techniques but it was also consistent with a higher incidence of the NTM in developed countries [3-7]. In Northern European countries, M. malmoense is actually one of the most prevalent nontuberculous pathogens $[1,3,6,8,9]$. Conversely, in the United Sates it has rarely been reported, and the majority of the isolates do not have a clinical relevance $[9,10]$.

M. malmoense infections are seen mainly in two age groups and with different clinical presentations. Most patients are male adults, with a mean age of 60 years, with underlying lung disease, especially chronic obstructive pulmonary disease (COPD), and present clinical symptoms and signs similar to those of pulmonary tuberculosis. The second group is formed by immunocompetent children, mostly younger than 5 years of age, with localized cervical lymphadenitis [1, 6, 8, 11-13]. Albeit rarely, other types of 
localized disease have been described, including skin, soft tissue infections and osteoarticular infections [14]. Disseminated disease is also remarkably rare and has been reported in patients with severely impaired immunity, except in one immunocompetent patient, described by Sarkar et al. [15], with pneumoconiosis and suspected of having liver and bone marrow dissemination.

We report a case of a 47-year-old immunocompetent man with a cervical abscess, in whom we identified a $M$. malmoense pulmonary disease with multiple cervical, thoracic and abdominal adenopathies.

\section{Case report}

A 47-year-old patient was sent to our Maxillo-Facial Surgery Department with a soft and non-adherent supraclavicular mass, with progressive enlargement for 2 months and without inflammatory signs (Fig. 1). The patient also complained of productive purulent cough for several months. There was no weight loss, fever or asthenia.

He had a medical history of left subclavian artery stenting in 2001, due to atherosclerotic stenosis. He smoked one pack of cigarettes a day since adolescence and had an alcohol consumption of 20-40 g/day. He was unemployed and denied recent medication, travel, previous tuberculosis or contact with a person diagnosed with tuberculosis.

Apart from the supraclavicular abscess, he had several small palpable masses on both sides of the neck, and a poor oral hygiene. No other signs were observed on the physical examination.

An ultrasound and a needle aspiration were performed and a head and neck computed tomography (CT) was requested. On ultrasound, a 6-cm multilocular cyst was seen, as well as multiple bilateral cervical enlarged lymph nodes.

On the following appointment, the cervical abscess had fistulized to the skin. We analyzed the head and neck CT that showed a hypodense lesion with $5 \mathrm{~cm}$ with peripheral

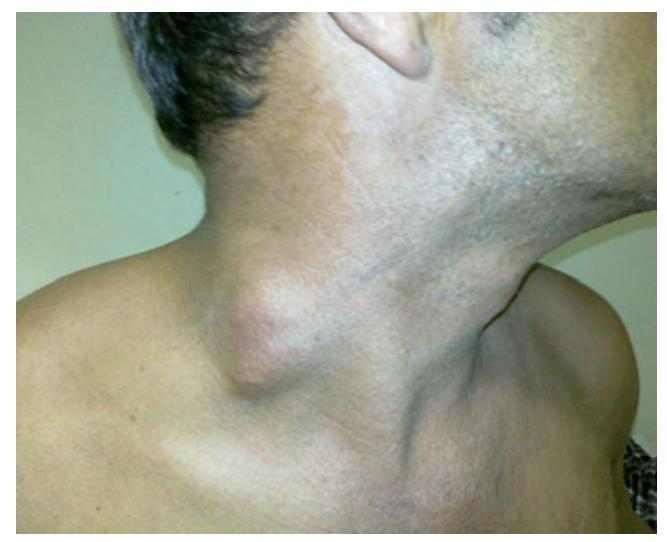

Fig. 1 Cervical abscess of the patient enhancement, multiple bilateral cervical lymphatic ganglions and a pulmonary cavitation on the right apex. The needle aspiration sample was negative for bacteria and fungi but inconclusive for mycobacteria. Due to the high suspicion of tuberculous infection, two ganglionar biopsies on both sides of the neck were performed, as well as a cutaneous excision of the fistulous tract.

The patient was then referred to the Infectious Diseases Department for further investigation, and started a treatment regime with isoniazid, ethambutol, rifampicin and pyrazinamide.

His chest X-ray demonstrated extensive bilateral nodular opacities on the apices. The high-resolution body CT performed showed multiple cavitations on both apices (the larger one was $6 \mathrm{~cm}$ ), signs of acute inflammatory process and multiple pulmonary, mediastinal and abdominal enlarged lymph nodes (Fig. 2). The blood tests showed a Creactive protein value of $5.06 \mathrm{mg} / \mathrm{dL}$ (normal $<0.5$ ), but were otherwise unremarkable, including for current HIV, hepatitis A, B and C virus, Cytomegalovirus, Epstein-Barr virus, Treponema pallidum, Brucella, Rickettsia conorii, Coxiella burnetii, Toxoplasma gondii, Mycoplasma pneumoniae, Legionella pneumophila and Bartonella infection, autoimmune serology, tumor markers and hormonal or protein electrophoresis changes. His blood and urine cultures were repeatedly negative. The tuberculin skin test was also negative. The biopsies of the cervical lymph nodes and the fistulized skin area showed multiple granulomatous inflammatory processes, and all were negative for ZiehlNeelsen, PAS and Grocott. The direct examinations and cultures of these samples were also unremarkable.

After 6 weeks of incubation, M. malmoense was identified on the sputum, bronchial aspiration and bron-

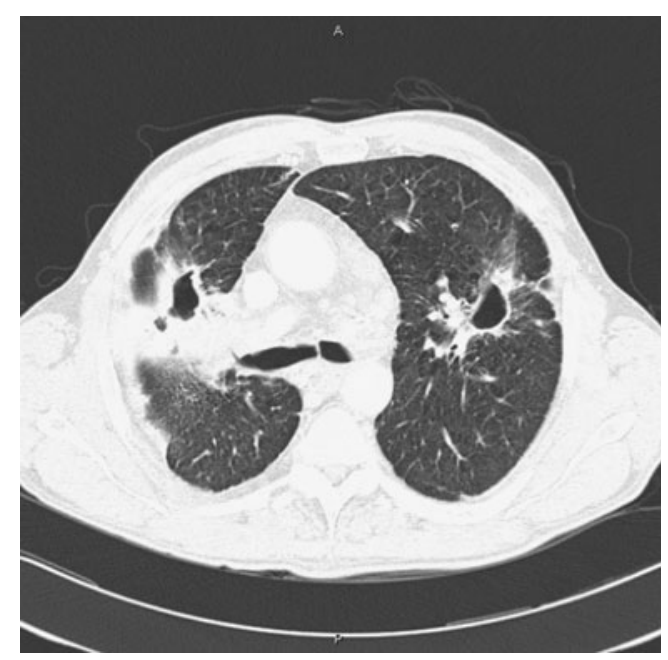

Fig. 2 Chest image of high-resolution computed tomography of the patient showing multiple cavitations on both upper lobes, signs of acute inflammatory process and distortion of the pulmonary parenchyma 
Table 1 American Thoracic Society diagnostic criteria for nontuberculous mycobacteria lung disease

Clinical criteria

1. Pulmonary symptoms, nodular or cavitary opacities on chest radiograph, or a high resolution CT scan that shows multifocal bronchiectasis with multiple small nodules; and

2. Appropriate exclusion of other diagnoses

Microbiological criteria

1. Positive culture results from at least two separate expectorated sputum samples; or

2. Positive cultures results from at least one bronchial wash or lavage; or

3. Transbronchial or other lung biopsy with mycobacterial histopathological features (granulomatous inflammation or acid-fast bacilli) and positive culture for NTM or biopsy showing mycobacterial histopathological features and one or more sputum or bronchial washings that culture positive for NTM

choalveolar lavage. The organism was isolated on MGIT ${ }^{\text {тM }}$ liquid cultures and identified by DNA sequence analysis with Inno-Lipa Mycobacteria $2^{\mathrm{TM}}$. No neoplastic cells were seen on the samples. After these results, pyrazinamide was withdrawn from the therapeutic regimen.

Two months after starting the medical treatment, a regression of the mass and the cervical lymph nodes was already seen as well as a remission of the purulent cough. Currently, at the sixth month of treatment, he is on a regimen containing isoniazid, ethambutol, rifampicin and clarithromycin with good tolerance and clinical evolution, and favorable prognosis.

\section{Discussion}

Pulmonary disease is the main manifestation of $M$. malmoense infection in adults and usually mimics pulmonary symptoms and radiological features of $M$. tuberculosis pulmonary disease $[1,16]$. The prevalence of pulmonary infection is higher in patients with pre-existing pulmonary disease, such as COPD, chronic bronchitis, emphysema, asthma, pneumoconiosis or healed tuberculosis [1].

According to the American Thoracic Society (ATS), our patient met the diagnostic criteria of the NTM lung disease, which distinguishes contamination or occasional presence of an NTM in a sample from a true pulmonary disease (Table 1) [9].

Although it is not possible to identify any mycobacteria from the neck samples in our patient, the histopathologic results of the cervical biopsies, showing multiple granulomatous processes, and the regression of the neck tumefactions after 2 months of treatment are consistent with a disseminated $M$. malmoense disease in an immunocompetent adult. In addition, according to the clinical investigation performed, no other current conditions responsible for cervical adenitis were identified, such as infectious, autoimmune, neoplastic or metabolic. Furthermore, we believe that $M$. malmoense infection could be responsible for all the other adenopathies identified on the body CT.

According to the ATS statement for NTM, even with excised nodes showing compatible histopathology, only $50-82 \%$ will yield positive cultures, especially for fastidious species [9]. As an example, a Canadian review of NTM adenitis cases over 10 years states a positive histopathology in $92 \%$ of the patients, but only $40 \%$ of the cases had positive cultures in the resected nodes [17]. These results should reinforce the importance of the index of suspicion to avoid diagnostic and treatment delays.

Upon review of the literature, cervical adenitis or abscesses due to M. malmoense in adults have been extremely rare. Our extensive search for similar clinical presentations on PubMed found five cases of cervicofacial manifestations for this pathogen in adults and, unfortunately, little was documented about their clinical history (Table 2). Only one case, described by Hoefsloot et al. [11], reported lymphadenitis concomitant to a pulmonary disease, but in a patient who received immunosupressive treatment after kidney transplantation.

Although cervical adenitis is the main extrapulmonary manifestation of $M$. malmoense, virtually all cases of cervical adenitis were documented in children. However, this presentation is still very uncommon when compared to the same disease due to M. avium complex [13]. It is argued by McCrossin and Mailman [18] that the occurrence of NTM adenitis in young children without predisposing conditions is perhaps a combination of immature immunity even if there is no evidence that they lack essential

Table 2 Cervical isolates of Mycobacterium malmoense in adult patients described on literature

\begin{tabular}{lllllll}
\hline Author, year & Country & Patients, $n$ & Sex & Age & Presentation & Associated pathologies \\
\hline Jenkins, 1985 [4] & United Kingdom & 1 & Female & 75 & Submandibular mass & Not specified \\
Henriques, 1994 [12] & Sweden & 1 & Female & 81 & Cervical abscess & Anaplastic thyroid cancer \\
Thomsen, 2002 [14] & Denmark & 1 & Male $^{\mathrm{a}}$ & $25^{\mathrm{a}}$ & Cervical $^{\mathrm{a}}$ & Not specified \\
Hoefsloot, 2009 [11] & Netherlands & 2 & Not specified & Elderly & Cervical lymphadenitis $^{\text {Not specified }}$ \\
\hline
\end{tabular}

${ }^{\text {a }}$ Personal communication 
immunological protection against mycobacteria as compared to adults [18] — and an increased exposure of their oral mucosa to environmental organisms due to less strict hygienic habits and pica [17]. Having this in mind, in our opinion, could be pertinent to consider that poor oral hygiene may also have a similar contribution to the etiology of the disease in adult patients. More recently, a genetic association study, involving 81 children with NTM cervicofacial lymphadenitis, hypothesized that children prone to NTM bear single nucleotide polymorphisms in genes that are relevant to mycobacteria immunity and associated with severity of periodontal disease - +3953TT in IL1B [18]. Clinical observations also suggest that vaccination with Bacille Calmette-Guérin (BCG) protects children against NTM lymphadenitis, according to the rates of the disease before and after the discontinuation of newborns' BCG vaccination in Sweden $[12,19]$. Focal cervical NTM disease when treated with complete surgical excision has a very high rate of success $[13,20]$. On the other hand, fine needle aspiration biopsy or incision and drainage of the involved lymph nodes without a complete excision may be followed by formation of sinus with chronic drainage, as happened in this case [9].

In contrast to localized neck NTM disease, in which a complete surgical resection is the most effective option, pulmonary and disseminated disease due to $M$. malmoense need a long-term regimen with antimicrobials. Based on randomized trials, the British Thoracic Society (BTS) recommends a 12- to 24-month regimen with rifampicin (RMP) and ethambutol (EMB) for pulmonary disease [8]. Although less tolerated and despite the lack of evidence of benefit to date, several authors, including the ATS statement on NTM, commented on the addition of isoniazid, ciprofloxacin or clarithromycin to the regimen with RMP and EMB [11, 21, 22]. Although in vitro tests suggested an increased susceptibility for a combination with clarithromycin [23] and some resistance to EMB and RMP, there is no evidence that drug susceptibility testing contributes to the choice and response of any treatment regimen $[1,8]$.

The mortality rate for M. malmoense pulmonary disease varied among different studies conducted in Europe, and difficulties in determining the precise cause of death may have influenced the results. In two studies, involving 18 and 30 patients, a $13 \%$ mortality rate was noted $[11,24]$. However, in a BTS trial [8] with 106 patients and, more recently, in a study conducted by Andréjak et al. [25] involving 46 individuals, a 5 -year mortality rate of $34 \%$ and $47.2 \%$, respectively, was observed. In two of these studies, the outcomes have been negatively influenced by comorbidities, involvement of more than one lung zone, the length of delay between the diagnosis and start of treatment, and, less strongly, EMB resistance $[8,11]$.

\section{Conclusion}

Extrapulmonary infection due to M. Malmoense, besides being a rare event, needs to be considered on the differential diagnosis of cervical masses and adenopathies, not only in pediatric patients but also in immunocompetent adults. In our opinion, this case report draws further attention to the emergence of $M$. malmoense as an infectious agent, considering its pathogenicity and the variability of clinical presentations among patients and among different parts of the world. Further investigation is needed to elucidate these issues.

For the diagnosis, it is essential to have a high index of suspicion for NTM disease in order to avoid diagnostic and treatment delays. Following appropriate laboratory procedures and recommendations, the identification of these fastidious agents using molecular probes is becoming more efficient. These instruments will be a fundamental aid in decreasing morbidity and mortality associated with the disease.

Acknowledgements We thank Rita Nobre Lucas, from Centro Hospitalar de Lisboa Central in Portugal, for technical and clinical expertise, and Vibeke Thomsen, from Statens Serum Institute in Denmark, for giving us further details about her article "Incidence and clinical significance of non-tuberculous mycobacteria isolated from clinical specimens during a 2-y nationwide survey".

Conflicts of interest The authors declare that there are no conflicts of interest.

\section{References}

1. Hoefsloot W, Boeree MJ, van Ingen J, Bendien S, Magis C, de Lange $\mathrm{W}$ et al (2008) The rising incidence and clinical relevance of Mycobacterium malmoense: a review of the literature. Int J Tuberc Lung Dis 12:987-993

2. Schröder KH, Juhlin I (1997) Mycobacterium malmoense sp. nov. Int J Syst Bacteriol 27:241-246

3. Martín-Casabona N, Bahrmand AR, Bennedsen J, Thomsen VO, Curcio M, Fauville-Dufaux M, Spanish Group for Non-Tuberculosis Mycobacteria et al (2004) Non-tuberculous mycobacteria: patterns of isolation. A multi-country retrospective survey. Int J Tuberc Lung Dis 8:1186-1193

4. Jenkins PA (1985) Mycobacterium malmoense. Tubercle 66:193-195

5. McGrath EE, Anderson PB (2007) Increased prevalence of nontuberculous mycobacteria infection. Lancet 370:28

6. Lamden K, Watson JM, Knerer G, Ryan MJ, Jenkins PA (1996) Opportunist mycobacteria in England and Wales: 1982 to 1994. Commun Dis Rep CDR Rev 6:R147-R151

7. Zaugg M, Salfinger M, Opravil M, Lüthy R (1993) Extrapulmonary and disseminated infections due to Mycobacterium malmoense: case report and review. Clin Infect Dis 16:540-549

8. Society BT (2003) Pulmonary disease caused by M. malmoense in HIV negative patients: 5-yr follow-up of patients receiving standardised treatment. Eur Respir J 21:478-482

9. Griffith DE, Aksamit T, Brown-Elliott BA, Catanzaro A, Daley C, Gordin F, ATS Mycobacterial Diseases Subcommittee, American Thoracic Society, Infectious Disease Society of America et al 
(2007) An official ATS/IDSA statement: diagnosis, treatment, and prevention of nontuberculous mycobacterial diseases. Am J Respir Crit Care Med 175:367-416

10. Buchholz UT, McNeil MM, Keyes LE, Good RC (1998) Mycobacterium malmoense infections in the United States, January 1993 through June 1995. Clin Infect Dis 27:551-558

11. Hoefsloot W, van Ingen J, de Lange WC, Dekhuijzen PN, Boeree MJ, van Soolingen D (2009) Clinical relevance of Mycobacterium malmoense isolation in The Netherlands. Eur Respir J 34:926-931

12. Henriques B, Hoffner SE, Petrini B, Juhlin I, Wåhlén P, Källenius G (1994) Infection with Mycobacterium malmoense in Sweden: report of 221 cases. Clin Infect Dis 18:596-600

13. Caruso G, Passàli FM, Salerni L, Molinaro G, Messina M (2009) Head and neck mycobacterial infections in pediatric patients. Int J Pediatr Otorhinolaryngol 73(Suppl 1):S38-S41

14. Thomsen VO, Andersen AB, Miörner H (2002) Incidence and clinical significance of non-tuberculous mycobacteria isolated from clinical specimens during a 2-y nationwide survey. Scand J Infect Dis 34:648-653

15. Sarkar A, Hussain RM, Aslam A, Peake MD (2001) Disseminated disease with Mycobacterium malmoense in a patient with pneumoconiosis. J Infect 43:215-216

16. Evans AJ, Crisp AJ, Colville A, Evans SA, Johnston ID (1993) Pulmonary infections caused by Mycobacterium malmoense and Mycobacterium tuberculosis: comparison of radiographic features. AJR Am J Roentgenol 161:733-737

17. McCrossin C, Mailman T (2006) First Canadian reports of cervical adenitis due to Mycobacterium malmoense and a 10year review of nontuberculous mycobacterial adenitis. Can J Infect Dis Med Microbiol 17:123-127

18. Haverkamp MH, Lindeboom JA, de Visser AW, Kremer D, Kuijpers TW, van de Vosse E et al (2010) Nontuberculous mycobacterial cervicofacial lymphadenitis in children from the multicenter, randomized, controlled trial in The Netherlands: relevance of polymorphisms in candidate host immunity genes. Int J Pediatr Otorhinolaryngol 74:752-754

19. Katila ML, Brander E, Backman A (1987) Neonatal BCG vaccination and mycobacterial cervical adenitis in childhood. Tubercle 68:291-296

20. Lindeboom JA, Kuijper EJ, Bruijnesteijn van Coppenraet ES, Lindeboom R, Prins JM (2007) Surgical excision versus antibiotic treatment for nontuberculous mycobacterial cervicofacial lymphadenitis in children: a multicenter, randomized, controlled trial. Clin Infect Dis 44:1057-1064

21. Banks J, Jenkins PA, Smith AP (1985) Pulmonary infection with Mycobacterium malmoense - a review of treatment and response. Tubercle 66:197-203

22. Jenkins PA, Campbell IA, Banks J, Gelder CM, Prescott RJ, Smith AP (2008) Clarithromycin vs ciprofloxacin as adjuncts to rifampicin and ethambutol in treating opportunist mycobacterial lung diseases and an assessment of Mycobacterium vaccae immunotherapy. Thorax 63:627-634

23. van Ingen $J$, van der Laan T, Dekhuijzen R, Boeree $M$, van Soolingen D (2010) In vitro drug susceptibility of 2275 clinical non-tuberculous Mycobacterium isolates of 49 species in The Netherlands. Int J Antimicrob Agents 35:169-173

24. Henry MT, Inamdar L, O'Riordain D, Schweiger M, Watson JP (2004) Nontuberculous mycobacteria in non-HIV patients: epidemiology, treatment and response. Eur Respir J 23:741746

25. Andréjak C, Thomsen VØ, Johansen IS, Riis A, Benfield TL, Duhaut P, Sørensen HT et al (2010) Nontuberculous pulmonary mycobacteriosis in Denmark: incidence and prognostic factors. Am J Respir Crit Care Med 181:514-521 